\title{
Up-Stream and Down-Stream Quality in Enterprise Modeling Practice - Supporting Model Driven Continuous Improvement in Organizations
}

\author{
Jarl Höglund ${ }^{1}$ and Anne Persson ${ }^{2}$ \\ ${ }^{1}$ ALLMENTOR AB, Storsvängen 66, SE-129 43 Mälarhöjden, Sweden \\ jarl.hoglundeallmentor.se \\ ${ }^{2}$ University of Skövde, Informatics Research Centre, P.O. Box 408, \\ SE-541 28 Skövde, Sweden \\ anne.persson@his.se
}

\begin{abstract}
The paper identifies and discusses what is considered to be the three most critical points, both up-stream and down-stream to Enterprise Modeling, for ensuring the usefulness and use of EM results in the continuous improvement of organizations. The model type that is targeted in the cases discussed in the paper is the process model, but other related model types, e.g. goal models and concepts models are also addressed in relation to process models. The points addressed in the paper are: 1) Trigger handling - acting on symptoms or the root cause problem, 2) critical aspects during modeling, and 3) establishing mechanisms for continuous model based business process improvement.
\end{abstract}

Keywords: Enterprise Modeling, process quality, successful implementation of enterprise models, continuous improvement.

\section{Introduction}

There are two main reasons for using Enterprise Modeling (EM) [1]:

- To develop the business. This entails developing business vision, strategies, redesigning the way the business operates, developing the supporting information systems, etc.

- To ensure the quality of the business. Here the focus is on two issues where EM can play an important part: (1) sharing the knowledge about the business, its vision and the way it operates, and (2) ensuring the acceptance of business decisions through committing the stakeholders to the decisions made.

In addition, EM can be used as a vehicle for communication and visualization in organizational problem solving.

Examples of EM methods can be found in [2, 3, 4, 5, 6 and 7]. Examples of application domains for EM can be found in [8, 9, 10, 11 and 12].

In order to achieve high quality results, the modeling process is equally important as the modeling language used. There are two levels of EM process:

The EM project level, where the modeling activities are placed in a context of purpose. [13] describes the generic process including the activities listed in Table 1. 
Table 1. Activities in EM [13]

Define scope and objectives of the modeling project
Plan for project activities and resources
Plan for modeling session
Gather and analyze background information
Interview modeling participants
Prepare modeling session
Conduct modeling session
Write meeting minutes
Analyze and refine models
Present the results to stakeholders

These activities can be categorized as belonging to two levels:

The modeling level where domain knowledge is gathered and enterprise models created and refined. When it comes to gathering domain knowledge to be included in enterprise models, the main EKD way of working is facilitated group sessions. In facilitated group session, participation is consensus-driven in the sense that domain stakeholders "own" the models and govern their contents. In contrast, consultative participation means that analysts create models and domain stakeholders are then consulted in order to validate the models. In the participatory approach stakeholders meet in modeling sessions, led by a facilitator, to create models collaboratively. In the sessions, models are often documented on large plastic sheets using paper cards. The "plastic wall" (Figure 2) is viewed as the official "minutes", for which every domain stakeholder in the session is responsible. There are two main arguments for using the participative approach, namely:

- The quality of models is enhanced if they result from collaboration between stakeholders, rather than from consultants' interpreting stakeholder interviews.

- The approach involves stakeholders in the decision making process, which facilitates the achievement of acceptance and commitment. This is particularly important when modeling is focused on changing some aspect of the domain, such as e.g. its visions/strategies, business processes and information system support.

In this paper we address these levels of the EM process, but we also take a step up from the process and puts this process in the context of continuous implementation of model-driven organizational improvements to achieve changing organizational goals. We call this the control level. The full EM lifecycle can be outlined as follows. It is also depicted in Figure 1.

1. Something triggers the need to investigate a potential change in the organization. This trigger can be a business opportunity, a challenge, a problem or a symptom of a problem. A choice is made to use EM in the investigation and potentially also to design a change to business operations.

2. The EM project is initiated and executed according to the process described in [13].

3. The implementation of the resulting models is planned and executed and the models now become part of the day-to-day business processes.

4. Continuous improvements are made. Some of these improvements could be supported by EM. Changes of greater importance will most likely cause the process to start over from 1 . 


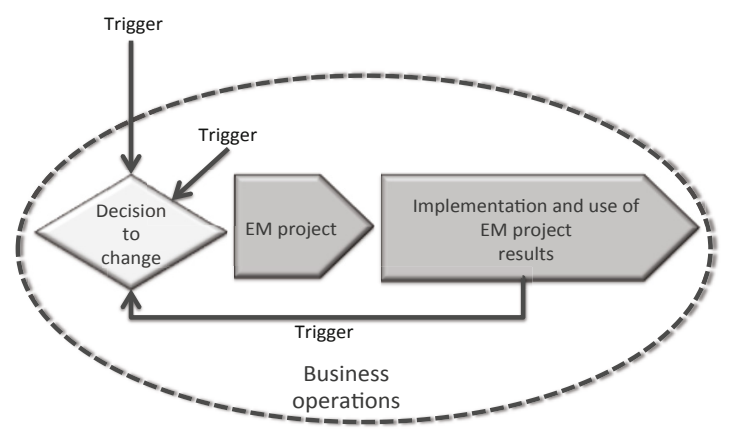

Fig. 1. EM in the context of continuous improvement

The outcome or effect of the implementation of models is very much dependent on

- How the EM project is planned and executed. From the point of view of the EM project we call this down-stream quality. Managing modeling and model quality is one aspect here as well as the many facets of managing the EM project as a whole.

- How the implementation and continuous improvement of the resulting models is planned and executed over time. From the point of view of the EM project we call this up-stream quality.

Their position in the continuous improvement process is depicted in Figure 2.

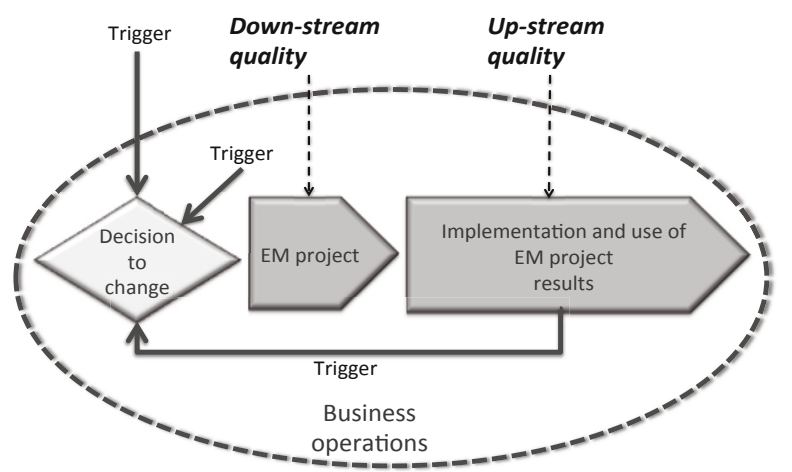

Fig. 2. Down-stream and up-stream quality in the context of continuous improvement

Effectively managing these two types of quality will ensure that the intended effect of modeling and the resulting models will materialize, not only from a short-term perspective but also long-term.

This paper is an experience paper that heavily relies on the experiences of the first author of the paper from 27 years of Enterprise Modeling and Enterprise Modeling method development. In fact, the first author was involved in developing the Swedish ABC EM method [14] that is the predecessor of both the Astrakan EM method ${ }^{1}$, that

\footnotetext{
${ }^{1}$ http: //www.astrakan.se/Om-Astrakan/Astrakanmetoden/ (in Swedish)
} 
has become quite a popular EM method in Sweden, and the Enterprise Knowledge Development (EKD) EM method [6]. He was also involved in developing participatory ways of working. The paper also builds on the experiences and research of the second author. She has, since the 1990-ies, specifically targeted the practice of EM in her research but has also substantial practical experience from EM projects. She has contributed to the development of the EKD EM method [6].

The goal of the paper is to identify and discuss what is considered to be the three most critical points, both up-stream and down-stream to EM, for ensuring the usefulness and use of EM results in continuous improvement of organizations. The model type that is targeted in the cases discussed in the paper is the process model, but other related model types, e.g. goal models and concepts models are also addressed in relation to process models. The points addressed in the paper are as follows:

- Trigger handling - acting on symptoms or the root cause problem.

- Critical aspects during modeling

- Establishing mechanisms for continuous model based business process improvement.

The remainder of the paper is organized as follows. Section 2 discusses the need to make decisions in starting an EM project that address root causes rather than symptoms. In section 3 some critical aspects in modeling are discussed, critical in the sense that we want to achieve organizational effects. The point of departure is process modeling. Section 4 focuses on how an effective mechanism for continuous organizational improvement can be implemented. Throughout the paper, illustrative examples from real life cases are provided. Finally, in Section 5, some concluding remarks are given.

\section{Trigger Handling - Acting on Symptoms or the Real Root Cause}

In order to ensure that the process of continuous improving organizational operations really serves the purpose of making the organization fit to take on its challenges and to prosper, it is essential that the organization acts on the "right" signals/triggers. This implies that the analysis of triggers needs to get enough and qualified attention, as a basis for making decisions about starting a change process. This will ensure that the EM project rests on firm ground from the start. We are simply starting the EM project for well-grounded reasons and with a goal that will effectively improve business operations. To illustrate this point, we describe two real life cases, one successful and one less successful.

\subsection{A Successful Case - Getting at the Root Causes}

\section{Situation}

A construction vehicle supplier with workshops for repair and maintenance all over Europe spends/wastes time and money. Some 5-10 administrators in each region are involved, on a daily basis to handle incorrect invoices, i.e. to credit and to correct 
failures. This is due to invoices stating the wrong amount and/or receiver. After correcting the errors, they finally send the correct invoices. Too many stakeholders are involved in this error correction process. This causes irritation and dissatisfaction for the involved stakeholders, but most serious is that no value is created.

\section{Action}

Nobody could state a "one and only" root cause to the problem, so a problem investigation was carried out. In order to involve the right participants a stakeholder diagram was made. A group of five prime and motivated members were selected and guided by a facilitator and they took on the task of carrying out the investigation.

\section{Method}

The method that was used was Root Cause Analysis (RCA) [15]. This is a method that has shown to be effective also to analyze the implications of new opportunities and threats, as an alternative to a SWOT analysis. The general principles of root cause analysis are as follows:

1. The primary aim of RCA is to identify the factors that resulted in the nature, the magnitude, the location, and the timing of the harmful outcomes (consequences) of one or more past events in order to identify what behaviors, actions, inactions, or conditions need to be changed to prevent recurrence of similar harmful outcomes and to identify the lessons to be learned to promote the achievement of better consequences. ("Success" is defined as the near-certain prevention of recurrence.)

2. To be effective, RCA must be performed systematically, usually as part of an investigation, with conclusions and root causes identified backed up by documented evidence. Usually a team effort is required.

3. There may be more than one root cause for an event or a problem, the difficult part is demonstrating the persistence and sustaining the effort required to develop them.

4. The purpose of identifying all solutions to a problem is to prevent recurrence at lowest cost in the simplest way. If there are alternatives that are equally effective, then the simplest or lowest cost approach is preferred.

5. Root causes identified depend on the way in which the problem or event is defined. Effective problem statements and event descriptions (as failures, for example) are helpful, or even required.

6. To be effective, the analysis should establish a sequence of events of timeline a to understand the relationships between contributory (causal) factors, root cause(s) and the defined problem or event to prevent in the future.

7. Root cause analysis can help to transform a reactive culture (that reacts to problems) into a forward-looking culture that solves problems before they occur or escalate. More importantly, it reduces the frequency of problems occurring over time within the environment where the RCA process is used.

8. RCA is a threat to many cultures and environments. Threats to cultures often met with resistance. There may be other forms of management support required to achieve RCA effectiveness and success. For example, a "non-punitory" policy towards problem identifiers may be required. 


\section{Outcome}

In contrast to the visible symptoms the following severe root causes resulted from the analysis. They were supported by hundreds of post-its on a big plastic folio on the wall, well sorted/related to each other:

1. The processes and their interfacing objects were not well defined. One aspect was mixed value-chains where, e.g., a vehicle all of a sudden became an invoice in the same process. Also, the input/output objects for steering and supporting processes were not satisfactorily specified, so in this case the workshop process was not quality controlled in its context.

2. At the customer reception the rules and policies to conform to, were too broad and complex and not easily accessible so the workshop receptionists hardly adhered to them.

3. The way to measure and reward responsible actors was in severe conflict, e.g. the workshop and service contract managers' KPIs were not aligned. Rather they were in conflict.

4. There was a lack of functionality and data accessibility in the IT support and there were also a variety of different systems in use. All in all, the IT support did not support the processes.

A number of opponent sessions according to the Astrakan EM method were then organized. In opponent sessions important stakeholders who have not been involved in creating the enterprise models are invited to discuss and criticize the models. This increased the quality of the analysis and created broad participation and commitment to invest and solve the key problems, i.e. it paved the way for next coming steps of successful improvement of the business operations. The four root causes became the basis to set a distinct purpose for next investigation project.

\subsection{A Less Successful Case - Acting on Symptoms}

\section{Situation}

A number of problems were experienced in the existing ordering process at an international high-tech company. The problems referred to were mainly overload, long lead-time and quality problems.

\section{Action}

No root cause analysis was made. The process was initially not described in relation to its context, so the interfaces to other processes were not included in the first analysis workshops.

The purpose of the first analysis was unclear, meaning that needed level of detail was not indicated. Substantial time and resources were spent. The project finally made a backward and forward analysis and found the sources of the main problems to be in processes before and after the ordering process that was initially the focus of the analysis. 
The assignment was expanded to include the whole process from Sales to Delivery, thus including the ordering process.

The analysis resulted in (1) demands on IT-projects in order to enhance the interfaces between current IT-systems and (2) make process interface agreements between the main processes.

\section{Method}

No method was really used from the start. Later the Astrakan EM method was used, both in order to identify and define the context of the process and to identify the process interfaces. Based on a concepts model, the terminology was aligned between the processes, thereby minimizing misunderstandings and as basis on which to update the requirements on data availability in the IT-support. However, by then the company had spent a large amount of resources and not getting anywhere in their efforts to solve the problem.

\subsection{Lessons Learned}

Already when we were 3-4 years old we stressed our parents by asking the question "WHY?" until we thought we had a sufficient answer, and then we were pleased. That behavior is natural for us as human beings and is one of the most important and easy to use "tools" in Root Cause Analysis and also in the Toyota Way, the LEAN Concept.

The main lessons that we learn from the above cases are:

- The situation that initially triggered the need for change must be clearly identified, and as soon as possible too, otherwise valuable time and resources will be wasted. Acting on symptoms as without asking the question why enough times is always bad.

- It is essential that the project manager or consultant involved arms herself/himself with sufficient arguments to justify why investments should be made in a Root Cause Analysis before the EM project is initiated and staffed.

- It is advisable to use a proven easy-to-use method, e.g. Root Cause Analysis (RCA). Free hands-on documentation about how to use the method is available on the web.

\section{$3 \quad$ Critical Aspects during Modeling}

Business/Enterprise Improvements are like making a journey or to go on an expedition. The better map you have the more pleasant and timely the journey or expedition will end. You will also know how to make the journey the next time, and you will have your eyes open for shortcuts or more scenic roads, i.e. added value. In EM a Process Model takes the role as the key map. It functions as the handrail where actors, information objects, rules/policies etc. can be attached in resource layers. Complementing models (e.g. goals models, concepts models and rules model) will be added depending on the cause and purpose of the improvement at hand. 


\subsection{Case 1 - A Systematic Way of Driving towards Improvement}

Yet another global company takes a strategic decision to sharpen a total solution concept and to increase the margins from the growing post market. The first steps were to analyze and describe a total solution sales process and to make a benefit analysis for the company and the customers. An EM project was started. All key roles were identified (project manager, business analyst, modeling facilitator, experts from the business operations). The key steps from a modeling point of view were:

1. Stakeholder analysis

2. SWOT analysis (not only filling the diagram, but to discuss and document conclusions which justified and updated the purpose of the investigation)

3. Modeling of the main process map using the Astrakan EM method was carried out in order to set the new sales process in its context.

a. The start and end of the process was identified.

b. Interface objects to/from the steering and supporting processes were identified as well as the interface to customers' purchasing process.

c. The future solution sales process was described using extensive interaction with stakeholder experts.

d. In resource layers rules/policies were mapped to the process as well as actors and interfaces to existing and required IT-support.

4. A benefit analysis proved the importance to start the most critical sub-projects for improving business operations suggested in the project report, which was based on the models.

This approach proved to pave the way towards successful implementation of change.

\subsection{Case 2 - Lack of Knowledge in the Organization about How to Run Improvement Projects}

This case involves an organization that manages a large pension fund. The main focus is on the back office function on which most other functions in the organization were dependent 24/7. Bad timing and bad quality in reports accelerated the stress on individual employees and on the function's interfaces, externally and internally.

An external consultant was assigned with the task to interview some stakeholders as a start. He indicated that the next coming step needed to be to do an initial investigation according to what was described in section 2.1 using EM. In parallel with the interviews the consultant started to make a stakeholder diagram and a main process map including interfaces to critical external actors. He paved the way for the next steps, but then something happened. When the interviews were made he was expected to give a full report of "what to do to solve the problem". The project was halted.

\subsection{Lessons Learned}

From the second case we can learn that it cannot be taken for granted that the problem owner/project initiator has insights in EM and is willing to carry out improvements 
accordingly. For internal as well as external consultants it is crucial not to start this type of investigation, or any EM activities for that matter, without double checking that the purpose is realistic and accepted, e.g. in terms of the way of working, resource availability, before starting the investigation. Make a plan and have it formally signed off!

If the organization, and particularly the problem owner, is not familiar with EM as a method an additional challenge is to "sell" the method to the organization and to get it accepted and sufficient resources allocated. The problem owner will always want to cut corners in terms of resources and to find shortcuts to what could be perceived to be a quick and solution. The quality of the outcome of EM will never benefit from this, and neither will the organization of course.

\section{Establishing Mechanisms for Continuous Model Based Process Improvement}

EM's moment of truth is when the future state process is implemented, a responsible process owner is in control, measurements are in place and used for continues follow up and rewards of process performance and continuous improvements. New opportunities and threats emanating from external or internal sources will challenge or ask for attention. Finding the complete toolbox to cope with continuous improvements is a never-ending process.

The quality of the map is crucial here, i.e. the process model and supporting models, which calls for quality assurance in all steps preceding the implemented and running process in its context.

Here we can find a positive case in Scania CV AB, Sweden, where continuous improvement is in "the spine" of all employees. Many academic papers have already been written referring to Scania and Continuous Improvements ${ }^{2}$, so we will not go into any detail about how this improvement process is organized.

What differentiates Scania in continuous improvements is perceived to be the following:

- $\quad$ The enterprise culture and leadership supports continuous improvement

- All employees are treated as experts in their role

- The personnel are loyal and proud of the company and their own work

- A problem is always turned into a possibility

\footnotetext{
${ }^{2}$ Examples of links to works on this topic are:

http: / / www . uppsatser. se/om/scania+st $\% \mathrm{C} 3 \% \mathrm{~A} 4$ ndiga+f $\% \mathrm{C} 3 \% \mathrm{~B} 6 \mathrm{rb} \% \mathrm{C} 3 \% \mathrm{~A} 4$ ttringar/ (mainly in Swedish)

http: //www.tandfonline.com/doi/abs/10.1080/00207540802538039

http://hig.diva-portal.org/smash/record.jsf?pid=diva2:126410

(In Swedish)

http: / / www. emeraldinsight. com/journals.htm?articleid=840538\&.sho $\mathrm{w}=\mathrm{abstract}$
} 
- Working in teams is encouraged

- "Right from me" is a well-established attitude in all personnel. This means that people make sure that what they deliver is correct and follow a high standard of quality.

- There is an established arena for dialogue/control/support, the "PULS" meetings, where all improvement activities are prioritized, are followed up, discussed, supported and made visible and thereby known and understood.

- $\quad$ There is an awareness of the fact that change may take time and is done in small controlled steps.

- There is a Scania Development Process supporting continuous improvements, where one of the initial phases is always Enterprise Modeling.

The effect of this is that the process of EM supported continuous improvement is kept alive and that external and internal triggers for change are properly analyzed and acted upon. Scania has proved to have a high degree of satisfied customers. The company has a high resilience in difficult times. E.g. in one of the latest financial crises many companies decided to lay off personnel. In Scania, on the other hand, the employees showed their loyalty by accepting a 4-day working week and a subsequent loss of salary of about $10 \%$. During the crisis the company involved their personnel in training activities. When the crisis decreased everyone was ready to start again but with an even better capacity.

\section{$5 \quad$ Concluding Remarks}

There has been much research into the quality of enterprise models (see e.g. [16]). However, more attention needs to be paid to the conditions under which models of high quality of created, as well as how the potential of implementing models can materialize in the context of continuous change.

In this paper we have identified critical quality aspects for EM to support continuous improvement process in an organization:

- Down-stream quality - to ensure that the EM project gets the best possible conditions. This includes:

○ Modeling quality - to ensure that modeling is carried out in the best possible way, and

O Model quality - to ensure that the resulting models are fit for purpose

- Up-stream quality - to ensure that the EM results effectively contribute to the continuous improvement process in an organization.

The paper provides a first starting point to looking at EM practice from an overall organizational level, particularly addressing continuous organizational improvements, and placing the EM project in that context. In this way, EM is addressed at three levels: 1) the control level, 2) the EM project level and 3) the modeling level.

This way of looking at the use and practice of EM could provide a framework for future research targeting more specific issues such as situational factors, goals of a 
modeling effort, goals for the desired model, process to be followed (a-priori method), actual process followed, conscious changes made to the a-priory process, tool usage, competency management, actual model quality, actual outcomes, actual use of the resulting models, etc.

The main limitation of this experience paper is that relies heavily for orientation and assumptions on the previous practice and research of its authors. We aim to extend the paper into a full research paper. This is why ongoing work involves comparing the experiences reported in the paper with relevant state of research papers.

\section{References}

[1] Persson, A., Stirna, J.: An explorative study into the influence of business goals on the practical use of Enterprise Modelling methods and tools. In: Tenth International Conference on Information Systems Development (ISD 2001), Royal Holloway, University of London, September 5-7 (2001)

[2] Bajec, M., Krisper, M.: A methodology and tool support for managing business rules in organisations. Information Systems 30(6), 423-443 (2005)

[3] Castro, J., Kolp, M., Mylopoulos, J.: A Requirements-Driven Development Methodology. In: Dittrich, K.R., Geppert, A., Norrie, M. (eds.) CAiSE 2001. LNCS, vol. 2068, pp. 108-123. Springer, Heidelberg (2001)

[4] Johannesson, P., Boman, M., Bubenko, J., Wangler, B.: Conceptual Modelling. Prentice Hall International Series in Computer Science, 280 pages. Prentice Hall (1997) Series editor Hoare, C.A.R.

[5] Willars, H., et al.: TRIAD Modelleringshandboken N 10:1-6 (in Swedish), SISU, Electrum 212, 16440 Kista, Sweden (1993)

[6] Bubenko Jr., J.A., Persson, A., Stirna, J.: User Guide of the Knowledge Management Approach Using Enterprise Knowledge Patterns, deliverable D3, IST Programme project Hypermedia and Pattern Based Knowledge Management for Smart Organisations, project no. IST-2000-28401, Royal Institute of Technology, Sweden (2001)

[7] Yu, E.S.K., Mylopoulos, J.: From E-R to "A-R" - Modelling Strategic Actor Relationships for Business Process Reengineering. In: Loucopoulos, P. (ed.) ER 1994. LNCS, vol. 881, pp. 548-565. Springer, Heidelberg (1994)

[8] Wangler, B., Persson, A., Söderström, E.: Enterprise Modeling for B2B integration. In: International Conference on Advances in Infrastructure for Electronic Business, Science, and Education on the Internet, L'Aquila, Italy, August 6-12. CD-ROM proceedings (2001)

[9] Wangler, B., Persson, A., Johannesson, P., Ekenberg, L.: Bridging High-level Enterprise Models to Implemenation-Oriented Models. In: Fujita, H., Johannesson, P. (eds.) New Trends in Software Methodologies, Tools and Techniques. IOS Press, Amsterdam (2003)

[10] Wangler, B., Persson, A.: Capturing Collective Intentionality in Software Development. In: Fujita, H., Johannesson, P. (eds.) New Trends in Software Methodologies, Tools and Techniques, pp. 262-270. IOS Press, Amsterdam (2002)

[11] Niehaves, B., Stirna, J.: Participative Enterprise Modelling for Balanced Scorecard Implementation. In: 14th European Conference on Information Systems (ECIS 2006), Gothberg, Sweden (2006) 
[12] Gustafsson, J., Höglund, J.: The Common Model of an Enterprise's Value Objects, Presented in Relevant Business Views. In: Persson, A., Stirna, J. (eds.) PoEM 2009. LNBIP, vol. 39, pp. 23-37. Springer, Heidelberg (2009)

[13] Stirna, J., Persson, A.: Purpose Driven Competency Planning for Enterprise Modeling Projects. In: Ralyté, J., Franch, X., Brinkkemper, S., Wrycza, S. (eds.) CAiSE 2012. LNCS, vol. 7328, pp. 662-677. Springer, Heidelberg (2012)

[14] Willars, H.: Handbok i ABC-metoden. Plandata Strategi (1988)

[15] Oaks, D.: Root Cause Analysis: the Core of Problem Solving and Corrective Action. ASQ Press (2009)

[16] Krogstie, J., Sindre, G., Jørgensen, H.: Process models representing knowledge for action: a revised quality framework. European Journal of Information Systems 15, 91-102 (2006) 